\title{
TELETERAPIA FONOAUDIOLÓGICA EN CONTEXTO EDUCATIVO CHILENO DURANTE LA PANDEMIA POR COVID-19 ${ }^{1}$
}

\author{
Marilyn San Martín², Yhara Gutiérrez ${ }^{3}$
}

\begin{abstract}
RESUMEN
Como consecuencia de la pandemia por covid-19 se ha debido implementar medidas como la suspensión de clases presenciales en las escuelas. La necesidad de adaptarse a esta emergencia global y la disponibilidad de herramientas tecnológicas ha permitido la activación de programas destinados a brindar opciones de atención terapéutica en contexto escolar, siendo la teleterapia uno de ellos. Este estudio busca describir el uso de teleterapia fonoaudiológica en educación regular durante la pandemia. Para esto se optó por un diseño tipo cuantitativo descriptivo, que permitió estudiar una muestra de 190 participantes de distintas regiones del país que cumplieron los criterios de elegibilidad, utilizando un cuestionario de Google para la recopilación de información. La información obtenida indica que la teleterapia se realizó con mayor frecuencia a través de sesiones sincrónicas y asincrónicas, de manera individual, una vez por semana y por 30 minutos. Asimismo, las dificultades relacionadas con la señal de internet y la prestación de insumos por parte del empleador fueron las principales barreras. Los resultados sugieren que la teleterapia puede ser una herramienta para dar continuidad a la intervención fonoaudiológica escolar, teniendo en cuenta que el covid-19 modificará radicalmente la prestación de servicios a mediano plazo.
\end{abstract}

Conceptos clave: educación regular, covid-19, teleterapia, fonoaudiología, trastornos de comunicación, telerehabilitación, telemedicina, trastornos del habla y lenguaje

\section{SPEECH AND LANGUAGE TELETHERAPY IN THE CHILEAN EDUCATIONAL CONTEXT DURING COVID-19 PANDEMIC}

\section{ABSTRACT}

As a consequence of the COVID-19 pandemic, measures such as the suspension of classes in schools have been implemented. The need to adapt arises with this global emergency and the availability of technological tools has allowed for the activation programs aimed at providing therapeutic care options in the school context, being teletherapy one of them. This study seeks to describe the use of speech-language pathology teletherapy in regular education during the pandemic. For this purpose, a quantitative descriptive-exploratory observational design was

1 Se le agradece a Poleth Corrotea Sanhueza, Estefanía Jara Unda, Yenifet Navarrete Zambrano, Valentina Palma Gutiérrez y Francisca Valdés Cofré (Estudiantes de Fonoaudiología, Universidad del Bío-Bío) por su colaboración y trabajo en el proceso de investigación de esta publicación, a todas gracias.

2 Universidad del Bío-Bío, Concepción, Chile. Contacto: msanmartin@ubiobio.cl

3 Universidad del Bío-Bío, Concepción, Chile. Contacto: ygutierrez@ubiobio.cl 
chosen. A sample of 190 participants from different regions of the country, who met the eligibility criteria, was studied, using a Google questionnaire to collect information. The information obtained indicates that teletherapy was most frequently performed through synchronous and asynchronous sessions, individually, once a week for 30 minutes. Likewise, difficulties related to the internet signal and the supply of material by the employer were the main barriers. The results suggest that teletherapy can be a tool to provide continuity to the school speech therapy intervention, taking into account that COVID-19 will radically modify the service delivery in the medium term.

Key concepts: regular education, covid-19, teletherapy, speech and language therapy, communication disorders, telerehabilitation, telehealth, speech-language pathology. 


\section{Introducción}

El sistema educativo chileno contempla diferentes apoyos a los estudiantes por medio del Programa de Integración Escolar (PIE), así como por la ley 20.248 Subvención Escolar Preferencial (SEP) (MINEDUC, 2008). Desde estos soportes el fonoaudiólogo centra su rol en el proceso terapéutico asociado con el lenguaje y la comunicación, y con todos los trastornos que en estas áreas se pueden presentar, por lo que se determina que es el profesional más indicado para intervenir sobre las necesidades educativas generadas a partir de estos déficits. Además, su participación es fundamental dentro del equipo colaborativo, ya que contribuye a abordar las necesidades de los estudiantes de manera integral (Vega, Torres y Del Campo, 2015), realizando evaluaciones e intervenciones en estudiantes con Necesidades Educativas Especiales (NEE), relacionadas principalmente con Trastornos Específicos del Lenguaje, y Trastornos de la Comunicación y/o Lenguaje asociados a patologías de base.

Si bien las NEE requieren de una intervención constante, existen ciertas instancias en las que surgen barreras para la continuidad terapéutica, tal como sucede en el contexto actual de nuestro país debido al impacto de la pandemia por covid-19. En el ámbito educativo, una de las medidas instauradas para la disminución de los contagios es el cierre de los establecimientos y la suspensión de clases presenciales. Según la UNESCO, este cierre afectó a 1.725 millones de niños y jóvenes (Eyzaguirre, Le Foulon y Salvatierra, 2020). Pese a ello, los profesionales de la educación intentaron mantener la continuidad del aprendizaje (OECD, 2020), lo que llevó a los fonoaudiólogos a buscar estrategias para el abordaje terapéutico no presencial, cobrando fuerza el concepto de "teleterapia", que subyace como una alternativa de reemplazo a las terapias presenciales. Según la Asociación Americana del Habla, Lenguaje y Audición, ASHA (2016), la teleterapia es el uso de la tecnología de las telecomunicaciones para servicios a distancia respecto de las diferentes áreas en las que se desempeña el profesional fonoaudiólogo, y permite desarrollar evaluación, intervención o generar consultas. Este es un método usado comúnmente en países 
desarrollados, en los que existe evidencia internacional de su uso e impacto positivo, ya que se considera una opción viable y eficaz para el tratamiento de niños con NEE, de fácil uso y confiable, tanto para padres como para terapeutas (Bradford et al., 2018).

Para llevar a cabo esta modalidad se establecen tres tipos de teleterapia: sincrónica, asincrónica e híbrida. La sincrónica corresponde a la interacción con un usuario o un grupo de personas, por medio de videos o audios en tiempo real, para lograr una experiencia similar a la tradicional. En el tipo asincrónico la información, ya sea imágenes, datos o actividades, es almacenada por el terapeuta y, posteriormente, enviada a los usuarios. Por último, en el híbrido se realiza una mezcla de lo asincrónico y lo sincrónico o presencial (ASHA, 2016).

El profesional que utiliza cualquiera de estas modalidades debe contar con una serie de condiciones que aseguren el resguardo tanto del usuario como de quien entrega la terapia. De esta forma deberá existir, entre otros aspectos, tecnología adecuada, registro actualizado de los números telefónicos de los estudiantes y un plan de emergencia ante cualquier eventualidad. Además, plena seguridad para la información audiovisual, el lugar debe ser privado y se debe obtener un consentimiento informado firmado por el usuario o cuidador (Cason y Cohn, 2014).

Aunque históricamente la mayor parte de la investigación sobre teleterapia logopédica se ha centrado en los servicios prestados a clientes adultos y en la evaluación de las habilidades de deglución, habla y lenguaje (Fairweather, Parkin \& Rozsa, 2004; Reynolds, Vick $\&$ Haak, 2009; Theodoros, 2008), Fairweather, Lincoln y Ramsden (2016) afirman que existe información que avala su uso. Edwards, Stredler-Brown y Todd (2012), en una revisión de la literatura sobre telepráctica en patologías del lenguaje y del habla, encontraron que la teleterapia es una forma eficaz de diagnosticar y tratar a niños y adultos, y que, en general, los servicios prestados en un entorno tradicional cara a cara o por teleterapia dieron resultados similares. Además, este tipo de servicio se ha utilizado en la evaluación y el tratamiento de una amplia gama de trastornos del lenguaje y del 
habla, incluidos los trastornos de la articulación, el autismo, la disartria, tartamudez, trastornos cognitivos comunicativos, disfagia y trastornos de la voz (Fairweather et al., 2016).

El estudio de Fairweather et al. (2016) recopiló información acerca de la teleterapia de niños que asistían a centros de atención temprana en zonas rurales, además de la opinión de sus padres, dando como resultado que su aplicación mediante videoconferencia mejoró las habilidades del lenguaje y del habla de los niños preescolares y escolares. Igualmente, las opiniones de los padres fueron positivas y la describieron como una modalidad más práctica, conveniente y eficiente, que permitía sesiones más frecuentes y consistentes. Pese a que la telepráctica parece ser una plataforma prometedora para brindar servicios de habla y lenguaje a niños en edad escolar, todavía no hay evidencia suficiente para confirmar que los resultados resultantes del tratamiento brindado a través de la teleterapia sean equivalentes a los del tratamiento en modalidad presencial (Rudolph $\&$ Rudolph, 2015).

Debido a esto, el objetivo de esta investigación es describir el uso de teleterapia fonoaudiológica en educación regular en tiempos de pandemia por covid-19, determinando las modalidades terapéuticas y las barreras teórico-prácticas de su aplicación.

\section{Método}

\subsection{Tipo de investigación, contexto y participantes}

Se escogió un diseño cuantitativo, descriptivo, utilizando un muestreo por conveniencia, fundamentado en la accesibilidad de los sujetos para el investigador (Otzen y Manterola, 2017). Se consideró para la muestra a fonoaudiólogos que implementaron teleterapia en educación regular en establecimientos municipales, particulares y subvencionados, urbanos o rurales a nivel nacional durante 2020. Para este efecto, se identificó a los participantes a través de correos electrónicos y redes sociales (Facebook e Instagram). La muestra estuvo conformada por 190 sujetos — 168 (88,4\%) de sexo femenino y $22(11,5 \%)$ de sexo masculino- pertenecientes a distintas regiones 
del país, en mayor porcentaje a la Región Metropolitana de Santiago y a la Región de Nuble (ambas con un 17,9\% de participación), concentrándose en un $80 \%$ en las zonas urbanas del país. De los participantes, $64(32,6 \%)$ tenían un rango de ejercicio profesional de uno a tres años y 11 meses, y 29 (15,2\%) con grado académico de Magíster.

\subsection{Técnicas de recopilación de información}

Se elaboró un cuestionario autorreportado, montado en la plataforma Google Forms. Las preguntas del cuestionario fueron elaboradas por el equipo de investigadores para dar respuesta a los objetivos de la investigación; con este fin se redactaron inicialmente 28 preguntas distribuidas en dos apartados relacionados con características sociodemográficas y laborales. De las 28 preguntas, cinco fueron de respuesta abierta y 23 de respuesta cerrada. A su vez, el cuestionario incorpora implícitamente dos ítems: el primero corresponde a "características sociodemográficas" y recopila datos como edad, sexo, nivel de estudio, rango de ejercicio profesional, ubicación regional de trabajo y zona a la que pertenece el establecimiento. El segundo a "características laborales" y encierra aspectos como tipo de establecimiento, jornada de trabajo, cantidad de estudiantes inscritos para atención fonoaudiológica, tipos de trastornos de lenguaje y comunicación que reciben terapia, cantidad de usuarios que reciben teleterapia, medios de comunicación utilizados, horas a la semana, tipo de actividades y su duración, frecuencia y tipo de atención de la teleterapia (grupal-individual-mixta), las actividades que se debe cumplir durante la pandemia en el proceso de intervención, los profesionales que participan en el trabajo colaborativo y sus respectivas horas dedicadas, frecuencia de comunicación, necesidades que han surgido de los apoderados, dificultades presentadas durante las sesiones, habilidades lingüísticas a trabajar e insumos proporcionados por el empleador.

El cuestionario fue sometido a juicio de expertos para su validación, con el propósito de obtener mayor objetividad y factibilidad de administración. Se contó con la colaboración de siete jueces expertos que cumplieron los criterios de elegibilidad de: a) 
tener título de fonoaudiólogo o educador diferencial, b) grado de Magíster en lenguaje y/o educación, y c) mínimo cinco años de experiencia en establecimientos educacionales. Cada uno de ellos analizó de forma independiente el cuestionario de acuerdo con dos indicadores específicos: "claridad" y "relevancia" (Escobar y Cuervos, 2008).

Los expertos realizaron comentarios y sugerencias, principalmente relacionadas con la redacción, y propusieron la incorporación de tres preguntas. A partir de las observaciones y aportes de los profesionales, el instrumento fue modificado y mejorado para su aplicación. Se envió nuevamente a los jueces expertos quienes aprobaron las 31 preguntas, obteniendo, a partir de los procedimientos anteriores, un instrumento titulado "Teleterapia fonoaudiológica en el ámbito educativo en tiempos de covid-19".

Consecuentemente, este cuestionario se utilizó para la recopilación de la información.

\subsection{Aspectos éticos}

Todos los participantes de la muestra (también los jueces que participaron en el proceso de validación por contenido del instrumento aplicado en este trabajo), fueron informados del estudio, sus objetivos, los procedimientos y el carácter voluntario y no remunerado de su participación, mediante un formato de consentimiento informado (artículo 11 de la Ley 20.120, 2006). Además, en este documento se garantizó que todas las actividades de colaboración estuvieran sujetas a la disponibilidad de tiempo de los participantes, pudiendo éstos abandonar el estudio sin dar razones que justificasen su decisión. También se informó que no existía riesgo como consecuencia de su participación. Además, se garantizó la confidencialidad de las opiniones que aportó cada juez experto (artículo 3 de la Ley 19.628, 1999). 


\section{Resultados}

Se estudió una muestra de 190 profesionales fonoaudiólogos de diferentes partes del país. En relación con aspectos sociodemográficos, el $88,4 \%$ de la muestra eran mujeres; el promedio de edad fue de 31,2 , con edades mínima y máxima de 23 y 50 respectivamente; el $36,8 \%$ refirió haber cursado pregrado, mientras que ninguno señaló haber cursado un doctorado; el 33,6\% de la muestra informó tener de uno a tres años de ejercicio profesional. El 17,9\% de los participantes se desempeña en las regiones de Nuble y Metropolitana; por otro lado, en la región de Aysén del General Carlos Ibáñez del Campo se encuentra un 0,5\% de la muestra; finalmente, el 80\% de los participantes pertenece a un establecimiento en zona urbana. El 50\% de los participantes trabaja en un establecimiento subvencionado o corporación; el 40\% de la muestra cuenta con jornadas laborales de 40 a 44 horas semanales (tabla 1 ).

Tabla 1.

Distribución de los participantes del estudio según características sociodemográficas

\begin{tabular}{lcc}
\hline \multicolumn{1}{c}{ Características sociodemográficas } & $\mathrm{n}$ & $\%$ \\
Sexo & 168 & 88,4 \\
Femenino & 22 & 11,5 \\
Masculino & 0 & 0 \\
Prefiere no responder & & \\
Edad (años) & & \\
Promedio: 31,2 (mínimo 23 máximo 50). & 62 & 32,6 \\
Nivel de estudio & 29 & 15,2 \\
Diplomados & 29 & 15,2 \\
Magíster & 70 & 36,8 \\
Postítulo & 0 & 0 \\
Pregrado & & \\
Doctorado & 64 & 33,6 \\
Rango de años de ejercicio profesional & 59 & 31,1 \\
1- 3 años 11 meses & 42 & 22,1 \\
4 - 6 años 11 meses & 25 & 13,1 \\
7- 9 años 11 meses & & \\
10 o más años & & \\
Ubicación regional de trabajo & 18 & 9,5 \\
Región de Valparaíso & 34 & 17,9 \\
Región Metropolitana de Santiago & 8 & 4,2 \\
Región del Libertador Bernardo O'Higgins &
\end{tabular}


Región del Maule 4,7

Región del Biobío 12,6

Región de Nuble 17,9

Región de la Araucanía

8,9

Región de los Lagos

5,3

Región de los Ríos

4,7

Región de Aysén del General Carlos Ibáñez del Campo

0,5

Región de la Antártica Chilena

Zona perteneciente del establecimiento

Rural

20,0

Urbano

80,0

Tipo de establecimiento

Municipal

$91 \quad 47,9$

Subvencionado o corporación

50,0

Particular

Jornada de trabajo

40 a 44 horas semanales

$\begin{array}{ll}76 & 40,0 \\ 53 & 27,9\end{array}$

30 a 39 horas semanales

20 a 29 horas semanales

18,4

10 a 19 horas semanales

35

10,0

9 o menos horas semanales

Las categorías diagnósticas mencionadas por los participantes fueron el Trastorno Específico del Lenguaje (TEL) expresivo y/o mixto (96,3\%), y el trastorno de la comunicación asociado a Trastorno del Espectro Autista (TEA) $(73,1 \%)$. El promedio de estudiantes inscritos para atención fonoaudiológica por establecimiento para 2020 fue de 48,7 y los que efectivamente recibieron teleterapia promediaron 28.

El 78,4\% de los participantes indicó que realizaba actividades mixtas, dedicando un promedio total de 33,9 horas. El 69\% de los profesionales realizaba sesiones semanales, con una duración promedio de 30 minutos en un 46,3\% de los entrevistados; el $56,3 \%$ indicó que la modalidad terapéutica fue predominantemente individual; los medios de comunicación más utilizados para las sesiones sincrónicas fueron las plataformas digitales (Zoom, Meet, Classroom, Moodle, Gennialy, Educaplay, Socrative, Menti, entre otras) en un 82,6\%. El tipo de material asincrónico enviado a los estudiantes corresponde en un $77,8 \%$ a guías y en un $76,8 \%$ a 
videos; estas actividades fueron monitoreadas posteriormente por los terapeutas en un $81,2 \%$ a través de WhatsApp, y en un 55\% por llamada telefónica; el 37\% indicó que la frecuencia de comunicación con los apoderados fue de solamente algunos días de la semana.

Entre las actividades ejecutadas por el fonoaudiólogo durante el teletrabajo se encuentran principalmente las reuniones con el equipo multidisciplinario, en un 95,3\%; terapia a través de videoconferencia, en un $91 \%$, y el envío de tareas fonoaudiológicas al hogar en un 86,3\%. El 56,3\% refirió trabajar primordialmente el nivel fonológico.

El promedio de horas de trabajo colaborativo fue de 9,2, sobresaliendo el trabajo colaborativo con el profesor/a de educación diferencial en un $87,4 \%$, con el psicólogo en un 58,94\% y con la profesora básica en un $58,4 \%$.

Tabla 2.

Modalidades terapéuticas utilizadas por los participantes

Modalidades terapéuticas

$\mathrm{n}$ $\%$

Registrados para atención fonoaudiológica 2020

Promedio: 48,7 .

Registrados para atención fonoaudiológica a través de teleterapia 2020 Promedio: 28.

Trastornos del lenguaje y la comunicación*

Trastorno específico del lenguaje expresivo y/o mixto 183

Trastorno de lenguaje asociado a discapacidad intelectual $\quad 120$

96,3

Trastorno de la comunicación asociado a TEA

63,1

Trastorno de la comunicación asociado a patología neurológica

Trastornos fonológico, fonético o mixto

Tipo de interacción

Sincrónicas (online)

Asincrónicas (offline)

Medios de comunicación sincrónica*

Llamada telefónica

Correo electrónico 
YouTube

Plataformas digitales

Monitoreo de material asincrónico*

Llamada telefónica

Correo electrónico

Videollamada

WhatsApp

Plataformas digitales

No se realiza monitoreo

Horas de teleterapia:

Promedio: 33,9.

Tipo de actividades asincrónicas enviadas *

Guías

Videos

PowerPoint

Plataformas digitales

Textos escolares digitales

Duración de sesiones sincrónicas

Menos de 30 minutos

30 minutos

46,3

45 minutos

33,6

Más de 45 minutos

Frecuencia de sesiones sincrónicas

Diaria

Semanal

Quincenal

Mensual

Metodología terapéutica

Grupal

Individual

Mixta

Actividades del fonoaudiólogo *

Terapia a través de videoconferencia

Reunión de apoderados

49,0

Jornadas de reflexión

60,0

Evaluaciones de proceso

68,0

Talleres o charlas dictadas o dirigidas por usted

46,0

Capacitaciones o perfeccionamientos

43,1

Turnos éticos

57,0

Evaluaciones presenciales cumpliendo las medidas sanitarias

Terapia presencial cumpliendo las medidas sanitarias 
Reunión con equipo multidisciplinario

Coenseñanza

Trabajo colaborativo*

Profesor/a de enseñanza básica

Profesor/a de enseñanza media

Educador/a de párvulos

Psicólogo

Terapeuta ocupacional

Profesor/a de educación diferencial

Trabajador social

Kinesiólogo

Jefe de Unidad Técnica Pedagógica o rol afín

No he realizado trabajo colaborativo

Frecuencia de comunicación apoderados

Todos los días, incluso fines de semana

Todos los días, excepto fines de semana

Solo algunos días de la semana

Un día a la semana

No hay comunicación con los apoderados

Habilidad lingüística abordada

Fonético

Fonológico

Semántico

Morfosintáctico

Pragmático

Discurso narrativo
181

76

95,3

40,0

111

58,4

13

117

112

52

166

39

30

95

7

40

29

7

47

4

17

107

28

17

6

15
6,8

61,6

58,94

27,3

87,4

20,5

15,8

50,0

3,7

21,0

15,3

3,7

25,0

2,1

8,9

56,3

15,0

9,0

3,2

7,9

Horas de trabajo colaborativo del fonoaudiólogo:

Promedio: 9,2.

*Variables con distribución de frecuencia de categorías.

La tabla 3 muestra la frecuencia de categorías de barreras teórico-prácticas mencionadas por los participantes. En ella se observa que, entre las opciones para dar continuidad al trabajo fonoaudiológico de estudiantes con dificultades de acceso a la teleterapia como alternativa, se utilizó en un 76,3\% el envío de material a través de medios digitales, en un $66,3 \%$ el retiro de material en el establecimiento y en un 42,6\% la entrega de material a domicilio. Los profesionales manifiestan que, entre las necesidades referidas por los apoderados, un 57,8\% afirmó presentar dudas acerca de las tareas fonoaudiológicas asincrónicas; un 57,3\% requerir apoyo para motivar a los estudiantes a participar, y un 36,8\% solicitó 
ayuda para el cumplimiento de los objetivos curriculares. Respecto de las dificultades durante la sesión, las principales quejas estaban relacionadas con la señal de internet, en un 91\%; con el compromiso de los apoderados, en un $72,1 \%$, y con la participación y motivación de los alumnos, en un 64,2\%.

Finalmente, en el ítem relacionado con la pregunta "su empleador ha proporcionado insumos para el trabajo a distancia requeridos por usted", el $41 \%$ manifestó que no habían sido proporcionados, un $29,5 \%$ afirmó que se les proporcionó material de oficina y solo un $16 \%$ reconoce haber recibido un computador.

Tabla 3.

Distribución de menciones de categorias sobre barreras teórico-prácticas

\begin{tabular}{|c|c|c|}
\hline Barreras teórico-prácticas & $\mathrm{n}$ & $\%$ \\
\hline \multicolumn{3}{|l|}{ Alternativas de contingencia } \\
\hline Retiro de material en el establecimiento & 126 & 66,3 \\
\hline Entrega de material a domicilio & 81 & 42,6 \\
\hline Envío de material a través de medios digitales & 145 & 76,3 \\
\hline Todos los estudiantes han podido acceder a las sesiones de teleterapia & 6 & 3,1 \\
\hline No se han realizado acciones & 6 & 3,1 \\
\hline \multicolumn{3}{|l|}{ Necesidades de apoderados } \\
\hline Dudas acerca de las tareas fonoaudiológicas sincrónicas & 67 & 35,2 \\
\hline Dudas acerca de las tareas fonoaudiológicas asincrónicas & 110 & 57,8 \\
\hline Solicitud de apoyo para el cumplimiento de los objetivos curriculares & 70 & 36,8 \\
\hline Dudas acerca de los avances en la terapia & 64 & 33,6 \\
\hline Necesidad de apoyo para motivar a los estudiantes a participar & 109 & 57,3 \\
\hline No han manifestado necesidades & 20 & 10,5 \\
\hline \multicolumn{3}{|l|}{ Dificultades en sesiones } \\
\hline Dificultades relacionadas con la señal de internet & 173 & 91,0 \\
\hline $\begin{array}{l}\text { Dificultades relacionadas con la participación y motivación de los } \\
\text { alumnos }\end{array}$ & 122 & 64,2 \\
\hline Dificultades en el uso de las plataformas & 91 & 47,9 \\
\hline Dificultades para entregar instrucciones y llevar a cabo la terapia & 51 & 26,8 \\
\hline Dificultades en el cumplimiento de horarios de las sesiones sincrónicas & 110 & 57,9 \\
\hline Dificultades relacionadas con el compromiso de los apoderados & 137 & 72,1 \\
\hline No ha tenido dificultades & 2 & 1,05 \\
\hline \multicolumn{3}{|l|}{ Insumos requeridos para el teletrabajo } \\
\hline Chip con conexión a internet & 23 & 12,1 \\
\hline Chip con minutos de telefonía & 6 & 3,2 \\
\hline Router & 4 & 2,1 \\
\hline Computador & 31 & 16,3 \\
\hline
\end{tabular}


Celular

$4 \quad 2,1$

Material de oficina

$56 \quad 29,5$

Suscripción a plataformas digitales

$31 \quad 16,3$

No le han proporcionado insumos

$78 \quad 41$

No ha requerido insumos

$20 \quad 11$

\section{Discusión}

Esta investigación tuvo como objetivo describir el uso de teleterapia fonoaudiológica en educación regular en tiempos de pandemia por covid-19, permitiendo determinar las modalidades terapéuticas utilizadas y las barreras teórico-prácticas para su implementación. Los resultados se obtuvieron a partir de una encuesta obtenida de un formulario de Google, el cual fue contestado por profesionales de diferentes regiones del país.

La metodología terapéutica más representativa estuvo relacionada con actividades sincrónicas realizadas por los fonoaudiólogos de manera individual con los estudiantes, resultados similares a los reportados por Grogan-Johnson, Alvarez, Rowan y Creaghead (2010), quienes concluyeron que las sesiones de terapia proporcionadas a través de la telemedicina fueron principalmente individuales.

En cuanto a los trastornos del lenguaje y de la comunicación, se observa en la muestra una mayor proporción de atención por telepráctica a niños con trastorno específico del lenguaje expresivo y/o mixto; de igual modo ocurrió con una muestra de 71 niños que recibieron teleterapia del habla y lenguaje, en la que un alto porcentaje pertenecía al diagnóstico de TEL (Gabel, Grogan-Johnson, Álvarez, Bechstein y Taylor, 2013). Si bien el TEL tiene una baja incidencia, tanto en Chile como en el extranjero (APA, 2002; CONADEE, 2014), los establecimientos educacionales con PIE atienden un alto número de estudiantes con alteraciones de este tipo (Holz, 2018).

En lo referente a la diversidad de actividades establecidas en los colegios para el cumplimiento de las funciones de los profesionales fonoaudiólogos durante la pandemia, destacan la terapia por videoconferencia, el envío de tareas fonoaudiológicas 
al hogar, reuniones con el equipo multidisciplinario y evaluaciones de proceso.

Respecto del último punto, los profesionales mencionan que realizaron procedimientos de evaluación por teleasistencia. Semel, Wiig y Secord (2006, citados en Gabel et al., 2013) investigaron a través de la cuarta edición de la Evaluación Clínica de los Fundamentos del Lenguaje (CELF-4) si podía administrarse con éxito cuatro de las subpruebas utilizando la telepráctica. Para este estudio participaron niños de cinco a nueve años, con desarrollo típico y sospecha de deterioro del lenguaje, los cuales fueron evaluados por fonoaudiólogos a través de telepráctica. Los investigadores concluyeron que el uso de la telepráctica para evaluar el lenguaje a través del CELF-4 es un método válido para realizar diagnóstico. Grogan-Johnson et al. (2010) exploraron, en las escuelas públicas rurales de Ohio, el uso de la telepráctica como modelo de prestación de servicio para estudiantes con trastornos de lenguaje y/o habla, en el que participaron treinta y cuatro estudiantes que recibieron la intervención, y por medio de un modelo directo aplicado en grupos pequeños y dirigido por un fonoaudiólogo. Los avances en el rendimiento se midieron mediante herramientas de seguimiento del progreso, incluidas las evaluaciones estandarizadas, la recopilación de datos y los informes trimestrales de avance que se suelen utilizar en el entorno de la escuela pública. Los resultados indican que los estudiantes progresaron en ambos modelos de prestación de servicios y que los avances fueron similares, independientemente del modelo utilizado. Si bien es viable realizar procedimientos de evaluación por teleasistencia, se debe avanzar en su seguimiento para valorar la confiabilidad, particularmente en nuestro país, por las baterías que usamos actualmente en el diagnóstico de los déficits.

En este estudio, la habilidad más trabajada fue el nivel fonológico, lo que es esperable debido a que estas dificultades en niños con TEL son un problema frecuente. Además, se acepta que los estudiantes que evidencian este cuadro pueden manifestar un desempeño descendido en la conciencia fonológica (Coloma y De Barbieri, 2007), lo que ha sido ratificado en numerosas investigaciones como la variable de mayor fuerza predictiva de la lectura inicial y 
posterior. Debido a esto, el entrenamiento en habilidades fonológicas mejora la ejecución en tareas de conciencia fonológica y facilita la adquisición de la lectura y la escritura (De la Calle, Aguilar y Navarro, 2016), por lo que se cree que se aborda mayoritariamente para dar respuesta a los objetivos curriculares y a las necesidades específicas de los estudiantes.

En relación con los tipos de actividades que se realizan en teleterapia, se observó que la mayoría era una mixtura de tareas sincrónicas y asincrónicas. En cuanto a lo sincrónico, lo más utilizado como medio de comunicación son las plataformas digitales, videollamadas y WhatsApp. En los estudios revisados se utilizan con mayor frecuencia las videoconferencias entre el usuario y el terapeuta (Fairweather, Lincoln \& Ramsden, 2017; Cason y Cohn, 2014; Gabel, et al., 2013). Fairweather et al. (2017) mencionan que las tecnologías más utilizadas para proporcionar el servicio sincrónico fue Adobe Connect, Facetime y Skype.

Acerca de los recursos digitales en modalidad asincrónica, los más usados por los profesionales encuestados fueron guías, videos y PowerPoint. De igual manera, para monitorear el avance de las tareas enviadas lo más utilizado fue WhatsApp y la llamada telefónica. En un estudio de teleterapia de patología del lenguaje y del habla en contexto educativo, los fonoaudiólogos trabajaron con niños con dificultades de comunicación utilizando documentos digitales, materiales pictóricos e interacciones verbales y no verbales. Para ello empleaban similares medios, como PowerPoint, PDF y juegos en línea, las cuales eran factibles de utilizar también al momento de compartir pantalla (Fairweather et al., 2016). Keck y Doarn (2014) concluyen que las actividades que incorporan tecnología de manera asincrónica para complementar la terapia son una solución favorable a las inestabilidades de conexión en tiempo real.

Las sesiones fueron semanales y su duración de 30 minutos, tiempo estimado que coincide con otros estudios de teleterapia ya publicados (Fairweather et al., 2016; Cason y Cohn, 2014; Gabel et al., 2013; Lincoln, Hines, Fairweather, Ramsden \& Martinovich, 2015). Además, se cree que la mayoría de los participantes usaba 
este tiempo, puesto que en el Decreto 170 el artículo 88 señala que la duración mínima de atención fonoaudiológica es de 30 minutos (MINEDUC, 2009).

Respecto del trabajo colaborativo, este se realiza principalmente con educadores diferenciales, así como con educadoras de párvulos, profesores de enseñanza básica y psicólogos, entendiendo que, tal como sostienen Torres y Montaña (2003), las relaciones entre los miembros de un grupo son el medio para fomentar el proceso enseñanza-aprendizaje. Según Peters (2002), su importancia se centra en potenciar el aprendizaje de los estudiantes, permitiendo su integración, el desarrollo del respeto, la comunicación y la toma de decisiones, con el propósito de intervenir significativamente en el usuario. Además, el trabajo en conjunto con los docentes involucrados en el proceso de aprendizaje se realiza para apoyar el desarrollo de los objetivos pedagógicos, los contenidos de las asignaturas y los logros y necesidades comunicativas de los estudiantes (Torres y Montaña, 2003).

En relación con las barreras teórico-prácticas, las más destacadas en la modalidad de teleterapia fueron los problemas de conexión a internet. Del mismo modo, en un estudio de descripción general de la teleterapia se mencionó que la velocidad de conexión y el ancho de banda afectaban considerablemente la calidad de la videoconferencia, interfiriendo en la fluidez de las sesiones (Cason y Cohn, 2014). En 2020, en Chile, según las estadísticas de la Subsecretaría de Telecomunicaciones, solo el 54,81\% de los hogares contaba con internet fijo. No obstante, de acuerdo con el ranking de reclamos en el mercado de telecomunicaciones, en el periodo 20192020 (2020) los reclamos recibidos fueron un total de 108.787, de los cuales el 52\% correspondía a la calidad técnica y/o a problemas del servicio.

Otra de las dificultades mencionadas, desde la perspectiva de los entrevistados, se relaciona con el compromiso de los apoderados durante las sesiones, lo que se contrasta con lo expresado por Fairweather, Lincoln y Ramsden (2017) quienes, al abordar patologías del lenguaje y el habla en el ámbito educativo, encontraron que, 
con frecuencia, en cada familia de los usuarios había un adulto que brindaba su apoyo en cada sesión, actuando como un miembro facilitador de la terapia. En efecto, este compromiso y apoyo familiar es sumamente importante. Si se introduce en el ámbito educativo se observa que, entre más próximos estén los apoderados, mayor es el impacto en el progreso del aprendizaje, participando de forma subyacente en actividades y tareas enviadas al hogar (Pizarro, Santana y Vial, 2013).

Cason y Cohn (2014) establecen la importancia de considerar en la selección del equipamiento el uso de un escritorio o de un sitio cómodo, un computador, tablet o teléfono, cámara web, micrófono accesorio en caso de ser necesario, y una buena capacidad de conexión a internet. A partir de lo anterior se determina que otra de las barreras está vinculada a la entrega y disposición de equipamiento e insumos necesarios para llevar cabo la teleterapia por videoconferencia, ya que los encuestados señalaron que sus empleadores no proporcionaron ningún tipo de ayuda al respecto, lo que se contrapone con el artículo No 152 del Código del Trabajo, que indica que será el empleador el encargado de suministrar el equipamiento necesario para llevar a cabo labores descritas en el contrato. Además, Gabel et al. (2013) mencionan que este tipo de equipamiento también debe ser proporcionado a los estudiantes por la institución a cargo de la teleterapia; no obstante, los profesionales encuestados indicaron que, para aquellos estudiantes a quienes se les dificultaba el ingreso a las sesiones, se optaba por la entrega de material digital para tareas asincrónicas o material impreso.

Finalmente, un estudio de telepráctica para la intervención escolar, comparó dos grupos de estudiantes con dificultades de habla y/o lenguaje: uno que recibía terapia de manera presencial y otro por teleterapia, concluyendo que existen muchas similitudes entre la modalidad presencial y la teleterapia, siendo esta última una opción viable y eficaz para la prestación de servicios de terapia del habla y de lenguaje. Así también demostró ser efectiva para la mayoría de los estudiantes que participaron en el estudio, ya que los avances tecnológicos permitieron tener buenos resultados en la prestación de servicios a distancia (Gabel et al., 2013). 


\section{Conclusiones y consideraciones finales}

Este estudio permitió cumplir con el objetivo inicialmente planteado: caracterizar la teleterapia con fonoaudiólogo en establecimientos educacionales, además de conocer la dedicación horaria en esta modalidad, tipos de diagnósticos abordados y las principales barreras y facilitadores. Esta información apoya la opinión de que la terapia proporcionada por medio de tecnologías puede satisfacer a los estudiantes y sus familias frente a la ausencia de terapia presencial en los establecimientos en época de pandemia. No obstante lo anterior, es necesario considerar que las familias no necesariamente poseen los elementos para apoyar y guiar el proceso formativo en el hogar por medio de la teleterapia, por lo tanto, entre los grandes desafíos de esta nueva modalidad se encuentra orientar y acompañar a las familias como coterapeutas, guiándolos en la utilización de nuevas herramientas, estrategias y formas de comunicación.

Por consiguiente, la teleterapia es una herramienta útil para la prestación de servicios fonoaudiológicos en funciones de promoción, prevención, evaluación, diagnóstico, intervención, asesoría y consejería, que genera alternativas viables de aplicación, extendiendo las posibilidades de ampliar el ejercicio de la profesión y mejorar las oportunidades de acceso a servicios educativos a distancia para promover la calidad de vida de los estudiantes en edad escolar. Sin bien este estudio informa resultados generales del uso de la teleterapia, es necesario considerar que es un modelo de prestación de servicios relativamente nuevo en nuestro país, por lo que faltan pruebas empíricas sobre la naturaleza, el alcance y la descripción de esta metodología. Se sugiere, por una parte, que los profesionales accedan a capacitaciones continuas y se conviertan en individuos competentes con la tecnología, conozcan las consideraciones éticas y resguarden la privacidad y seguridad de los estudiantes; por otra, tener en cuenta factores como los costos, la disponibilidad de recursos y las necesidades de diagnóstico e intervención de los estudiantes, así como comprender que el nuevo papel de la tecnología en el diagnóstico y tratamiento de los trastornos de la comunicación es vital para la expansión de la teleterapia como estándar de atención, con el fin de lograr el desarrollo de normas y directrices técnicas. 
Finalmente, para futuras investigaciones se propone incluir en el abordaje de esta temática la percepción de los estudiantes y apoderados sobre el proceso de terapia a distancia, pues son ellos quienes deberían informar su grado de satisfacción con esta nueva modalidad. Así también se propone que se siga caracterizando la teleterapia, con el propósito de incorporarla de manera cotidiana como una modalidad válida y eficaz, que permita complementar la terapia presencial en Chile, o ser una alternativa a esta cuando, por razones geográficas, económicas o de salud, los estudiantes no puedan acceder a terapia con la manera tradicional.

\section{Referencias}

Asociación Americana de Psiquiatría (APA). (2002). Manual Diagnóstico y Estadístico de los Trastornos Mentales DSM-IV-TR. Barcelona: Masson.

Asociación Americana del Habla, Lenguaje y Audición (ASHA). (2016). Telepráctica. Recuperado el 10 de Julio 2020 de https://www.asha. org/practice-portal/professional-issues/telepractice/

Bradford, N., Caffery, L., Taylor, M., Meiklejohn, J., Smith, A. y Langbecker, D. (2018). Servicios de patología del habla y lenguaje prestados por telesalud en un entorno educativo rural: la perspectiva de la escuela. Revista de la Sociedad Internacional de Telemedicina y E. Health, 6(1), e20, 1-8. DOI: https://doi.org/10.29086/JISfTeH.6.e20

Bravo, L. (2004). La conciencia fonológica como un posible "zona de desarrollo próximo" para el aprendizaje de la lectura inicial. Revista Latinoamericana de Psicología, 36, 21-32.

Cason, J. y Cohn, E. (2014). Telepráctica: descripción general y mejores prácticas. Perspectivas sobre la comunicación aumentativa y alternativa, 23(1), 4. DOI: 10.1044/aac23.1.4.

Coloma Tirapegui C. J. y De Barbieri Ortiz, Z. (2007). Trastorno fonológico y conciencia fonológica en preescolares con trastorno específico del lenguaje. Revista de Logopedia, Foniatría y Audiología, 27(2), 67-73.

CONADEE, Educación especial Chile. (2014). Estudio preliminar de las prevalencias proyectadas de las necesidades educativas especiales asociadas o no a discapacidad, en el sistema educacional chileno. Santiago: CONADEE, Mesa Técnica.

De la Calle, A., Aguilar, M. y Navarro, J. (2016). Desarrollo evolutivo de la conciencia fonológica: ¿Cómo se relaciona con la competencia lectora posterior? Revista de Investigación en Logopedia, 1, 22-41. 
Edwards, M., Stredler-Brown, A. \& Houston, K. T. (2012). Expanding Use of Telepractice in Speech-Language Pathology and Audiology. The Volta Review, 112(3), 227-242.

Escobar, J. y Cuervo, A. (2008). Validez de contenido y juicio de expertos: una aproximación a su utilización. Avances de Medición, 6, 35.

Eyzaguirre, S., Le Foulon, C. y Salvatierra, V. (2020). Educación en tiempos de pandemia: antecedentes y recomendaciones para la discusión en Chile. Estudios Públicos, (159), 111-180.

Fairweather, C., Parkin, M. \& Rozsa, M. (2004). Speech and language assessment in school-aged children via videoconferencing. In B. E. Murdoch, J. Goozee, B. Whelan y K. Docking (Eds.), Speech Pathology Australia. Retrieved at https://www.telemedecine-360.com/wp-content/ uploads/2019/02/2015-SPA0113_Position_Statement_Telepractice_ in_Speech.pdf

Fairweather, G. C., Lincoln, M. A. \& Ramsden, R. (2016). Speech-language pathology teletherapy in rural and remote educational settings: Decreasing service inequities. International Journal of Speech-Language Pathology, 18(6), 592-602. DOI: 10.3109/17549507.2016.1143973

Fairweather, G. C., Lincoln, M. A. \& Ramsden, R. (2017). Speech-language pathology telehealth in rural and remote schools: the experience of school executive and therapy assistants. Rural and Remote Health, $17(3), 4225$.

Gabel, R., Grogan-Johnson, S., Álvarez, R., Bechstein, L. \& Taylor, J. (2013). A Field Study of Telepractice for School Intervention Using the ASH NOMS K-12 Database. Communication Disorders Quarterly, 35(1): 4453. DOI: $10.1177 / 1525740113503035$

Grogan-Johnson, S., Alvarez, R., Rowan, L. \& Creaghead, N. (2010). A pilot study comparing the effectiveness of speech language therapy provided by telemedicine with conventional on-site therapy. Journal of Telemedicine and Telecare, 16, 134-139.

Holz, M. (2018). Datos de la modalidad de Educación Especial en Chile. Recuperado el 18 de mayo de 2021 de https://obtienearchivo.bcn. cl/obtienearchivo?id=repositorio/10221/26781/2/BCN__datos_de_ EE_y_estudiantes_con_NEE_Final.pdf

Keck, C. S. \& Doarn, C. R. (2014). Telehealth Technology Applications in Speech-Language Pathology. Telemedicine and e-Health, 20(7), 653659. DOI: 10.1089/tmj.2013.0295

Lincoln, M., Hines, M., Fairweather, C., Ramsden, R. \& Martino Vich, J. (2014). Multiple stakeholder perspectives on teletherapy delivery of 
speech pathology services in rural schools: A preliminary, qualitative investigation. International Journal of Telerehabilitation, 6, 65-74.

Ministerio de Educación, MINEDUC. (1 de febrero de 2008). Ley 20.248 de subvención especial preferencial. Recuperado de https://www.bcn.cl/ leychile/navegar?idNorma $=269001$

Ministerio de Educación, MINEDUC. (14 de mayo de 2009). Decreto con toma de razón $\mathrm{N}^{\circ}$ 170. Fija normas para determinar los alumnos con necesidades educativas especiales que serán beneficiarios de las subvenciones para educación especial. Santiago de Chile: Mineduc.

OECD. (2020). El impacto de COVID-19 en educación, perspectivas de educación en un vistazo. Paris: OECD Indicadores, OECD Publishing. DOI: http:// dx.doi.org/10.1787/eag-data-en.

Otzen, T. y Manterola, C. (2017). Técnicas de muestreo sobre una población a estudio. Int. J. Morphol., 35(1), 227-232.

Owens, R. (2003). Desarrollo del lenguaje. Pearson Education.

Peters, O. (2002). La educación a distancia en transición. México: Universidad de Guadalajara.

Pizarro, P., Santana, A. y Vial, B. (2013). La participación de la familia y su vinculación en los procesos de aprendizaje de los niños y niñas en contextos escolares. Diversitas: Perspectivas en Psicología, 9(2), 271287.

Reynolds, A. L., Vick, J. L. \& Haak, N. J. (2009). Telehealth applications in speech-language pathology: A modified narrative review. Journal of Telemedicine and Telecare, 15(6), 310-316.

Rudolph, J. M. \& Rudolph, S. (2015). Telepractice vs. On-Site Treatment: Are Outcomes Equivalent for School-Age Children. EBP Briefs, 10(2), $1-15$.

Semel, E., Wiig, E. H. \& Secord, W. A. (2006). Clinical evaluation of language fundamentals (4th ed., CELF-4). Toronto, Canada: The Psychological Corporation/A Harcourt Assessment Company.

SERNAC. (2020). Ranking de Reclamos en Mercado Telecomunicaciones 20192020. SERNAC: Información de mercados y productos. Recuperado de https://www.sernac.cl/portal/619/w3-article-62164.htm

Theodoros, D., Hill, A., Russell, T., Ward, E. \& Wootton, R. (2008). Assessing acquired language disorders in adults via the Internet. Telemedicine and e-Health, 14, 552-559.

Torres, S y Montaña, M., (2003). El modelo colaborativo. Una alternativa para el trabajo del fonoaudiólogo en el sector educativo. Revista 
Areté, 3(1), 31-37. Recuperado de https://arete.ibero.edu.co/article/ view/574

Vega, Y., Torres, A. y Del Campo, M., (2015). Autorreflexión sobre el desempeño profesional del fonoaudiólogo(a) en el sector educativo: una aproximación a la construcción del rol. Revista Chilena de Fonoaudiología, 14, 103-117.

Recibido: 20/05/2021

Aceptado: 14/12/2021 